\title{
The role of the resonant "stickiness" in the dynamical evolution of Jupiter family comets
}

\author{
A. Alvarez-Candal and F. Roig \\ Observatório Nacional, Rua Gal. José Cristino 77, 20921-400, Rio de Janeiro, RJ, Brazil. \\ email: alvarez@on.br
}

\begin{abstract}
We analyze the effect of the temporary capture of comet-like orbits in asteroid mean motion resonance by following the dynamical evolution of 2090 Jupiter-family cometlike orbits over $10^{7} \mathrm{yr}$ under the perturbation of the four major planets. The resonant capture may be related to the phenomenon known as "resonant stickiness" consisting in the temporary stabilization of very eccentric orbits near the separatrices of the mean motion resonances. We found that the population of orbits that were captured at least once during the simulation has a median lifetime larger than that of the complete sample.
\end{abstract}

Keywords. Celestial Mechanics, comets: general.

\section{Introduction}

The resonance stickiness arises when a very eccentric orbit becomes temporarily captured near the separatrix of a mean motion resonance. The "sticky" orbits are chaotic although practically stable, having dynamical lifetimes longer than we should expect from their short Lyapunov times. These orbits usually evolve confined in a limited region of the phase space (Murison, Lecar, and Franklin, 1994).

In the asteroid belt the resonant stickiness has been identified as being responsible for the "stable chaos" (Milani and Nobili, 1992; Milani, Nobili and Knežević, 1997) detected in some high-order mean motion resonances (Tsiganis, Varvoglis, and Hadjidemetriou, 2000; 2002). On the other hand, Malyshkin and Tremaine (1999) found that the rate of decay of the population of ecliptic comets, and in particular of the Jupiter-family comets (JFC), is mostly explained by considering stickiness phenomena of the orbits near resonant islands in the phase space.

In this contribution, we analyzed the dynamical evolution of a sample of JFC-like orbits, aiming to study the effect of the resonance stickiness in their dynamical lifetime.

\subsection{The numerical setup}

From a list of cometary nuclear magnitudes provided by G. Tancredi (pers. comm.), we selected 190 known JFC with orbital elements referred to JD 2452700.5. Then, we generated a set of JFC test orbits by cloning each real orbit ten times. The orbital elements of these clones were chosen at random in the intervals $\Delta a= \pm 0.1 \mathrm{AU}, \Delta I=$ $\pm 1.5^{\circ}$, and $\Delta M= \pm 10^{\circ}$ around the reference orbit ( $M$ denotes the mean anomaly). The eccentricity was cloned so as to preserve the value of the Tisserand parameter (with respect to Jupiter) of the reference orbit.

The dynamical evolution of this set of 2090 test particles (real JFC plus clones) was followed over $10^{7}$ yr using the symplectic integrator SWIFT RMVS3 (Levison and Duncan, 1994) and taking into account the perturbation of the four major planets. The integration time-step was 1.8 days, and the simulation of a particle was interrupted whenever: (i) it 
came closer than 0.01 Hill radius to some planet, (ii) it collided with the Sun, or (iii) its heliocentric distance became larger than $200 \mathrm{AU}$.

\section{Results}

Figure 1 shows the depletion of the entire set of test orbits over time. The median lifetime of the set is $\tau=1.7 \times 10^{5} \mathrm{yr}$, which is about half the value estimated by Levison and Duncan (1994). This discrepancy may be due to the different model used by these authors, or to the much smaller sample of test orbits simulated by them.

A two-stages behavior is evident from Fig. 1. Before $\tau_{s} \sim 3 \times 10^{5} \mathrm{yr}$, the population decays following an exponential law $\left(N_{1}(t) \propto e^{\left(-4 \times 10^{-6} t\right)}\right)$. After $\tau_{s}$, the decay switches to a power law $\left(N_{2}(t) \propto t^{-1.104}\right)$. This kind of behavior has been previously reported by Kerney (1983), Levison and Duncan (1994) and Malyshkin and Tremaine (1999). The exponential decay is expected from a random-walk behavior induced by close encounters with the planets and also from the existence of hyperbolic regions of the phase-space, devoided of stability islands. The switch to a power law decay indicates that some mechanism or mechanisms are delaying the depletion of orbits. This is usually interpreted as a consequence of the resonance stickiness, and the time $\tau_{s}$ is usually referred to as the "stickiness time".

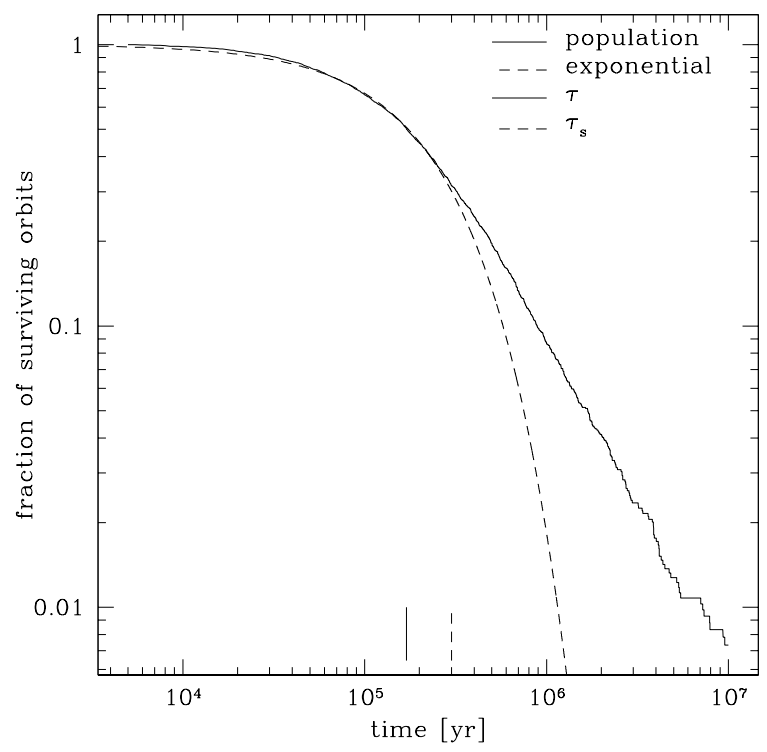

Figure 1. The decay of our set of test orbits, normalized to the total number of initial orbits.

To analyze the resonant capture of test orbits during the simulation, we first computed the mean orbital elements of the orbits by running averages of the osculating elements. We applied a running window 10,000 yr wide, and mean elements were determined every 500 yr. Then, we considered that an orbit suffered a resonant capture if, at any moment during the simulation, it had mean semi-major axis $\bar{a}$ satisfying the condition $\left|\bar{a}-a_{\text {res }}\right|<$ $\Delta a_{\text {res }}$ over at least 10,000 yr. The nominal semi-major axes, $a_{\text {res }}$, of the considered resonances as well as their corresponding widths, $\Delta a_{r e s}$, are shown in Table 1 . We found that 304 test particles from our sample became temporarily captured in any of these resonances. This number does not account for the test orbits that were already captured 
Table 1. Nominal semi-major axes and corresponding widths of the analyzed resonances. The resonance width was defined as half the minimum distance to the adjacent resonances. The last column indicates the total number of captured orbits.

\begin{tabular}{cccc|cccc}
\hline $\mathrm{p}: \mathrm{q}$ & $a_{\text {res }}[\mathrm{AU}]$ & $\Delta a_{\text {res }}[\mathrm{AU}]$ & $\mathrm{N}$ & $\mathrm{p}: \mathrm{q}$ & $a_{\text {res }}[\mathrm{AU}]$ & $\Delta a_{\text {res }}[\mathrm{AU}]$ & $\mathrm{N}$ \\
\hline $8: 3$ & 2.704602 & 0.06 & 24 & $7: 5$ & 4.155888 & 0.025 & 19 \\
$5: 2$ & 2.823509 & 0.06 & 21 & $4: 3$ & 4.293289 & 0.055 & 35 \\
$7: 3$ & 2.956410 & 0.035 & 33 & $9: 7$ & 4.398652 & 0.04 & 9 \\
$9: 4$ & 3.028965 & 0.035 & 52 & $5: 4$ & 4.482042 & 0.04 & 29 \\
$2: 1$ & 3.276392 & 0.125 & 36 & $6: 5$ & 4.605694 & 0.045 & 23 \\
$9: 5$ & 3.514802 & 0.035 & 28 & $7: 6$ & 4.693009 & 0.035 & 12 \\
$7: 4$ & 3.581436 & 0.03 & 26 & $8: 7$ & 4.757965 & 0.025 & 2 \\
$5: 3$ & 3.699844 & 0.055 & 48 & $9: 8$ & 4.808182 & 0.02 & 3 \\
$8: 5$ & 3.801917 & 0.05 & 34 & $10: 9$ & 4.848167 & 0.02 & 3 \\
$3: 2$ & 3.969067 & 0.07 & 37 & $1: 1$ & 5.200949 & 0.18 & 112 \\
$10: 7$ & 4.100290 & 0.03 & 18 & & & & \\
\hline
\end{tabular}

from the very beginning of the simulation (77 orbits). Moreover, in order to safely avoid any possible bias caused by the cloning process, we did not take into account for tests orbits that became captured in any of these resonances during the first 30,000 yr of the simulation either.

Analyzing this sub-population of captured orbits, we found that their residence times (defined as the time interval for which the orbit remains captured in the resonance) are generally shorter than $10^{5} \mathrm{yr}$. However, in some particular cases, as for example the 7:4 resonance, it may be as long as $10^{6} \mathrm{yr}$ (Fig. 2). We also found that the sub-population of captured orbits has a median lifetime which is twice that of the entire population (Fig. 3).

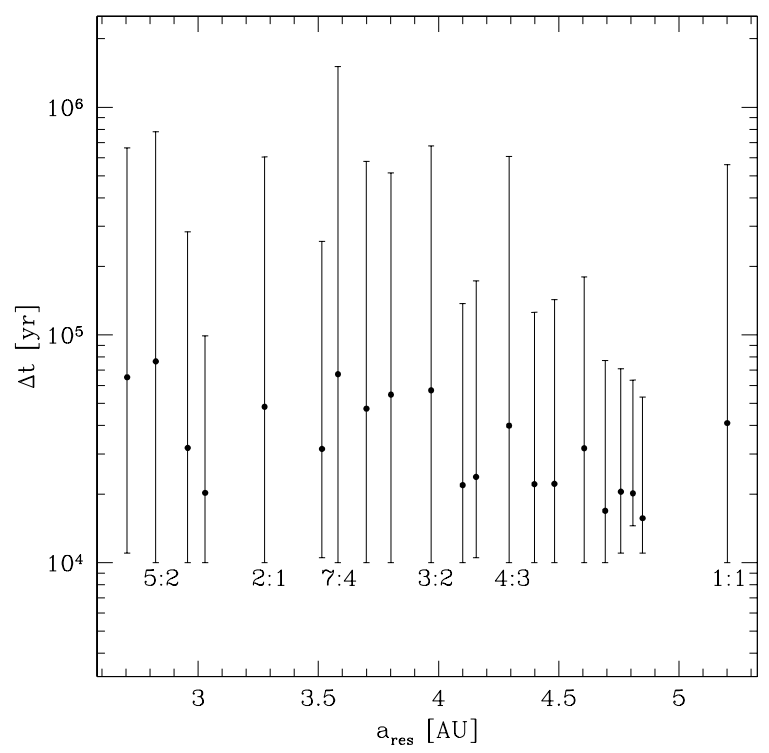

Figure 2. Mean residence times in the analyzed resonances. The minimum and maximum residence times are indicated by the error bars. 


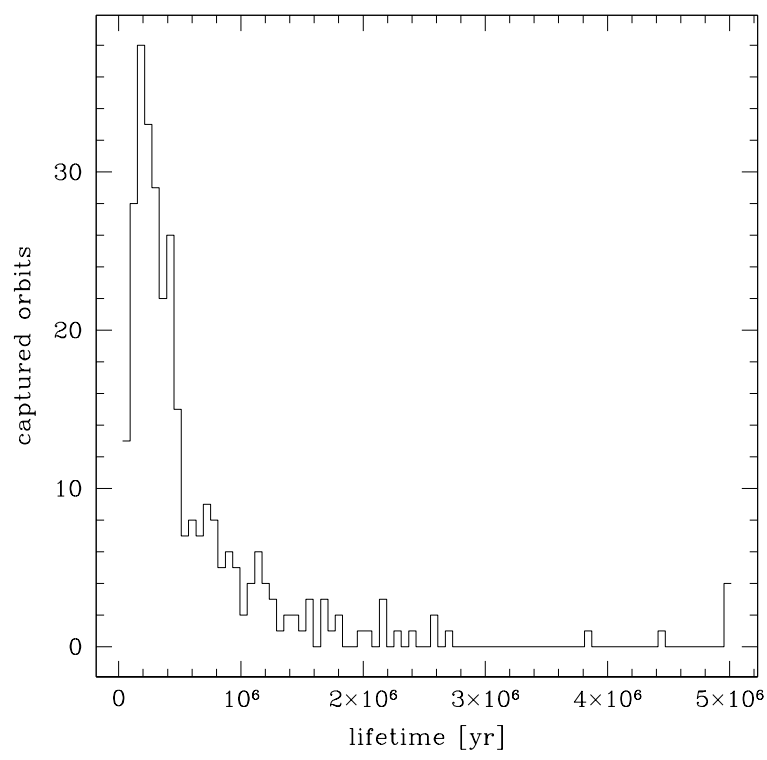

Figure 3. The dynamical lifetimes of the orbits that became captured in any resonance during the simulation, distributed in bins of $60,000 \mathrm{yr}$. The median lifetime is $\tau \sim 3.6 \times 10^{5} \mathrm{yr}$.

\section{Conclusions}

According to our simulations, the population of resonant captured orbits has a median lifetime longer than the complete sample, which seems to support the hypothesis of the resonance stickiness as the mechanism which temporarily stabilizes cometary orbits in mean motion resonances. We must recall that we are talking here about "long-term" resonant captures, with residence times of the order of $10^{4}-10^{5} \mathrm{yr}$. These must be distinguished from the "short-term" captures reported by Carusi et al. (1985) which last for only a few hundred years. The existence of these long-term captures may imply that a non negligible fraction of the known resonant asteroids evolving in chaotic orbits could actually be JFCs. This puts interesting constraints to the search of possible extinct cometary nuclei evolving on highly eccentric NEAs orbits or on asteroid-like orbits in the outer belt. However, these results are very preliminar and a better analysis of the data is required before drawing definite conclusions.

\section{Acknowledgements}

This work has been supported by CNPq and CAPES.

\section{References}

Carusi, A., Kresak, L., Perozzi, E., \& Valsechi, G.B. 1985, Long term evolution of short-period comets, Adam Hilger, Bristol

Karney, C.F.F. 1983, Physica D 8, 360

Levison, H. \& Duncan, M. 1994, Icarus 104, 18

Malyshkin, L. \& Tremaine, S 1999, Icarus 141. 341

Milani, A. \& Nobili, A. 1992, Nature 357, 569

Milani, A., Nobili, A., \& Knežević, Z 1997, Icarus 125, 13

Murison, M., Lecar, M., \& Franklin, F. 1994, Astron. J. 108, 2323

Tsiganis, K., Varvoglis, H., \& Hadjidemetriou, J. 2000, Icarus 146, 240

Tsiganis, K., Varvoglis, H., \& Hadjidemetriou, J. 2002, Icarus 155, 454 